\title{
Interference alignment for a multi-user SISO interference channel
}

\author{
Yasser Fadlallah*, Karine Amis, Abdeldjalil Aïssa-El-Bey and Ramesh Pyndiah
}

\begin{abstract}
Our work addresses the single-input single-output interference channel. The goal is to show that although interference alignment is suboptimal in the finite power region, it is able to achieve a significant overall throughput. We investigate the interference alignment scheme proposed by Choi et al. (IEEE Commun. Lett. 13(11): 847-849, 2009), which achieves a higher multiplexing gain at any given signal dimension than the scheme proposed by Cadambe and Jafar (IEEE Trans. Inform. Theory 54(8), 2008). Then, we try to modify the IA design in order to achieve enhanced sum-rate performance in the practical signal-to-noise ratio (SNR) region. Firstly, we introduce a way to optimize the precoding subspaces at all transmitters, exploiting the fact that channel matrices in the interference model of a single-input single-output channel are diagonal. Secondly, we propose to optimize jointly the set of precoder bases within their associated precoding subspaces. To this end, we combine each precoder with a new combination precoder, and this latter seeks the optimal basis that maximizes the network sum rate. We also introduce an improved closed-form interference alignment scheme that performs close to the other proposed schemes.
\end{abstract}

Keywords: Interference channel; Single input single output; Optimization problem; Precoding; Interference alignment

\section{Introduction}

In most existing wireless multi-user communication systems, interference is avoided either by coordinating the users to orthogonalize the channel access or by treating interference from other transmitters as noise. However, until recently, the capacity region of the interference channel (IC) remained unknown, except for some special cases such as strong and very strong interference [1,2]. In [3], Maddah-ali et al. have proposed a new approach in order to show that the $N$-antennas MIMO X channels can offer as much as $\frac{4 N}{3}$ degrees of freedom (DoF). This new approach of interference management has been named IA.

The key idea of IA is to jointly design all transmitted signals such that interfering signals at each receiver overlap and remain distinct from the desired signal. This approach has been exploited by Cadambe and Jafar in [4]. The authors have shown that the maximum achievable DoF in the $K$-user time-varying single-input singleoutput (SISO) IC, in the $n$ dimensional Euclidean space,

\footnotetext{
*Correspondence: yasser.fadlallah@telecom-bretagne.eu Institut Télécom; Télécom Bretagne; UMR CNRS 6285 Lab-STICC, Technopôle Brest Iroise CS 83818, Brest 29238, France
}

is $\frac{K}{2}$ and is achieved, thanks to an IA scheme. Later on, Motahari et al. have addressed the achievable DoF of a quasi-static IC. They have extended the idea of IA from space/time/frequency dimensions to the signal level dimensions and have shown that based on the field of Diophantine approximation in number theory [5], the interference can be aligned in the rational spaces, achieving a maximum DoF of $\frac{K}{2}$.

The first IA scheme for SISO transmissions has been proposed in [4] for the time/frequency-varying channel. This scheme has been designed to achieve the asymptotic capacity in the IC, i.e., when both the signal-tonoise (SNR) and the signal dimensions tend to infinity. In contrast, Choi et al. have introduced another IA design that aims to achieve a higher multiplexing gain at any given signal dimension [6]. In this paper, we adopt an IA scheme for SISO transmission, and we try to modify the design in order to achieve higher sumrate performance in the practical SNR region. Most references, among which [7-9], deal with IA schemes for MIMO interference channels. However, all mobile communication standards still include a SISO transmission

\section{Springer}

(c) 2014 Fadlallah et al: licensee Springer. This is an Open Access article distributed under the terms of the Creative Commons Attribution License (http://creativecommons.org/licenses/by/2.0), which permits unrestricted use, distribution, and reproduction in any medium, provided the original work is properly credited. 
mode as for instance the LTE downlink transmission mode 1 . This is the reason why in this paper, we try to define efficient IA schemes for SISO interference channels.

In our contributions, we firstly introduce a way to optimize the precoding subspaces at all transmitters, exploiting the fact that channel matrices in the IA model are diagonal. Two solutions are derived; the first is achieved iteratively using projected gradient descent method, the second is a closed-form solution that avoids the numerical computation, thus, resulting in a very low computational complexity. Secondly, we propose to optimize the precoding vectors at each transmitter within its precoding subspace. To this end, we combine each IA precoder with a new combination precoder. The combination precoder seeks the optimal basis that maximizes the network sumrate assuming an individual transmit power constraint. However, a closed-form solution does not seem trivial. Therefore, we apply an iterative process based on the simple gradient descent method, which converges to a local maximum due to the non-concavity of the objective function.

This paper is organized as follows. Section 2 describes the system model. Then, Section 3 presents the IA design in SISO IC. In Section 4, we propose to optimize the network sum rate through a diagonal matrix $W$. The precoding vector optimization within the IA subspaces is presented in Section 5. In Section 6, we present the convergence rate of the proposed iterative algorithms. Section 7 evaluates the sum-rate performance of the proposed optimization. Finally, Section 8 concludes the paper.

Notations: boldface upper case letters and boldface lower case letters denote matrices and vectors, respectively. For the transpose, transpose conjugate, and conjugate matrices, we use (.) ${ }^{T},(.)^{H}$, and (.)* respectively. $\boldsymbol{I}_{p}$ is the $p \times p$ identity matrix, and $\mathbf{1}_{p}$ is the all-one vector of length $p$.

\section{System model}

Let us assume a $K$-user SISO IC with $K$ transmitterreceiver pairs. A wireless channel links each receiver to each transmitter, but a given transmitter intends to have its signal decoded by a single dedicated receiver only. Let us denote by $s_{j}$ the length $d_{j}$ symbol vector to be transmitted by the $j$ th user. As shown in Figure $1, s_{j}$ is precoded by applying an $N \times d_{j}$ precoding matrix yielding the length $N$ vector $\boldsymbol{x}_{j}$. We assume a frequency-selective channel, and the inter-symbol interference is perfectly managed using an orthogonal frequency division multiplexing (OFDM) transmission scheme. The number of subcarriers is equal to $N$, and all components of $\boldsymbol{x}_{j}$ are simultaneously transmitted on a one-component-per-subcarrier basis. The received signal at the $k$ th receiver can be modeled as

$$
\boldsymbol{y}_{k}=\sum_{j=1}^{K} \boldsymbol{H}_{k j} \boldsymbol{V}_{j} \boldsymbol{x}_{j}+\boldsymbol{z}_{k}, \forall k \in \mathcal{K},
$$

where $\mathcal{K}=\{1, \cdots, K\}$ is the set of all users, $\boldsymbol{H}_{k j} \in \mathbb{C}^{N \times N}$ is the diagonal channel matrix between the $j$ th transmitter and the $k$ th receiver, $V_{j} \in \mathbb{C}^{N \times d_{j}}$ is the precoding matrix of the $j$ th transmitter. The $j$ th transmitted information $\boldsymbol{x}_{j}$ is defined as a $d_{j} \times 1$ vector belonging to a Gaussian continuous constellation. $z_{k}$ is the $N \times 1$ circularly symmetric complex Gaussian noise vector at the receiver $k$, with independent and identically distributed (i.i.d.) components, i.e. $z_{k} \sim \mathcal{N}_{c}\left(0, \sigma^{2} \boldsymbol{I}_{N}\right)$. We also consider the following hypotheses in this paper:

1. Users do not cooperate.

2. Non-precoded user symbols are Gaussian continuously distributed and mutually independent.

3. The set of channel matrices $\boldsymbol{H}_{k j}$ is entirely and perfectly known at all transmitters and all receivers.

4. All diagonal components of $\boldsymbol{H}_{k j} \forall k, j \in \mathcal{K}$ are i.i.d. and continuously distributed, with absolute values upper-bounded with a finite value.

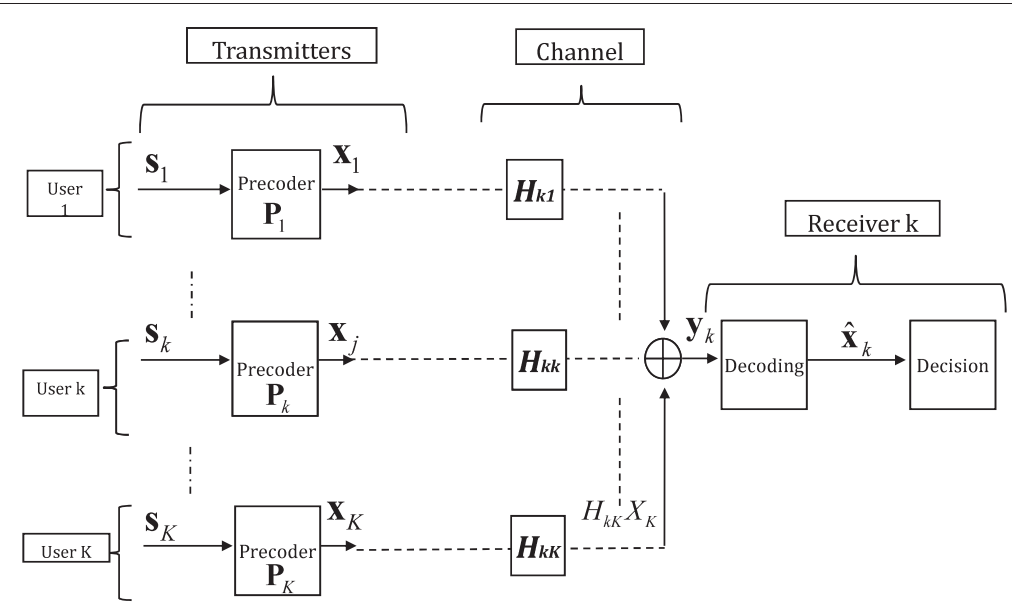

Figure 1 Interference channel model. 
The maximum achievable DoF in the $K$-user SISO IC is equal to $[4,5]$

$$
\lim _{\mathrm{sn} r \rightarrow \infty} \frac{C(\mathrm{sn} r)}{\log _{2}(\mathrm{sn} r)}=\frac{K}{2},
$$

where $C(\mathrm{snr})$ represents the channel capacity.

\section{IA design in a SISO interference channel \\ 3.1 Precoding design}

The essence of the IA scheme is to design the transmit beamforming matrices in a way that the interference-free stream number at each receiver is maximized. The IA design conditions have been defined as follows:

$$
\begin{array}{r}
\operatorname{rank}\left(\boldsymbol{U}_{k} \boldsymbol{H}_{k k} \boldsymbol{V}_{k}\right)=d_{k}, \\
\boldsymbol{U}_{k} \boldsymbol{H}_{k j} \boldsymbol{V}_{j}=0, \forall j \neq k,
\end{array}
$$

where $\boldsymbol{U}_{k}$ is the decoding matrix at the $k$ th receiver. In other words, the desired signal belongs to the subspace generated by the vectors of $\boldsymbol{G}_{k}=\boldsymbol{U}_{k} \boldsymbol{H}_{k k} \boldsymbol{V}_{k}$, while the interference is completely eliminated. The feasibility of the linear system in (3) is conditioned to the following proprieties: (i) the linear system has to be proper, i.e., the number of variables is more than or equal to the number of equations and (ii) the linear system has to be generic [10]. In some particular cases, the genericity is satisfied by providing a channel matrix with random and independent coefficients.

Figure 2 illustrates the IA principle in the case of three users sharing the same resources to communicate. Each transmitter has one symbol to transmit to its dedicated receiver. All channel coefficients are supposed to be known at all transmitters ${ }^{\mathrm{a}}$. In order to achieve the IA linear precoding design, each transmitter $k$ transmits its symbol in the direction of a two-dimensional vector $\boldsymbol{v}_{k}$, and the precoding vectors at all transmitters are designed in such a way that at all receivers the vectors carrying the two interfering symbols are aligned and linearly independent of the vector that carries the desired symbol. Then, the interference is eliminated by a simple projection on the interference null space.

One precoding design that provides IA at all receiver nodes and fulfills the conditions in (3) in the SISO interference channel is proposed by Choi et al. as [6]

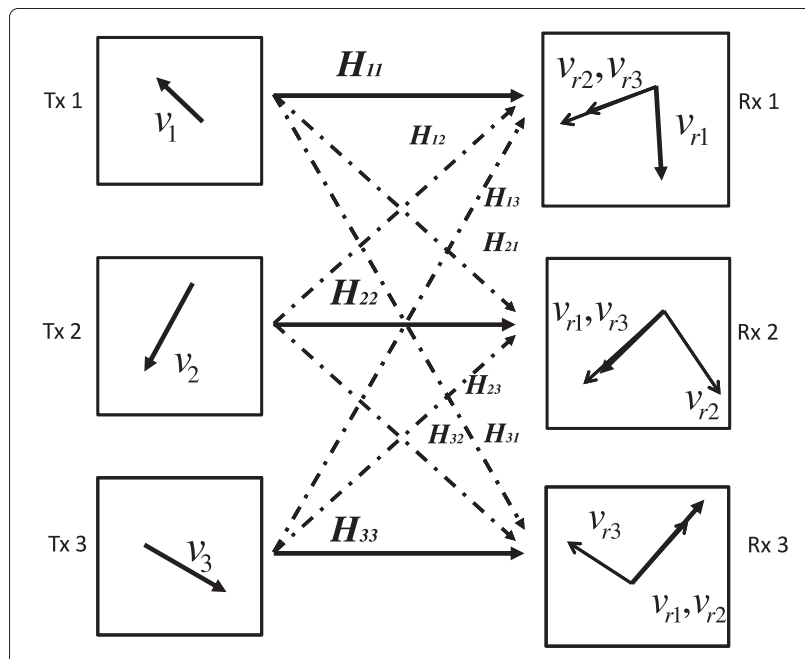

Figure 2 Three-user SISO interference channel with IA scheme.

where $m^{*}$ is any non-negative integer which defines the number of transmitted symbols and the length of the precoding vectors, and $\boldsymbol{T}_{k l}$ is an $N \times N$ diagonal matrix. In the IA design described previously, the achievable DoF per user can be obtained using the following combinations:

$$
d_{1}=\left(\begin{array}{c}
m^{*}+M+1 \\
M
\end{array}\right) \text { and } d_{3}=\left(\begin{array}{c}
m^{*}+M \\
M
\end{array}\right)
$$

where $M$ is a parameter depending on the user number, $M=(K-1)(K-2)-1, d_{i}$ is the DoF of the $i$ th user, i.e., the number of transmitted symbols, and $N$ the number of symbols in each IA vector is defined as $N=d_{1}+d_{2}$. In the particular scheme previously, the IA conditions can be satisfied by providing $d_{i}=d_{3}, d_{1}>d_{3}, i \in \mathcal{K} \backslash\{1,3\}$. For example, in a three-user SISO multi-user IC, we have $d_{1}=n+1, d_{2}=d_{3}=n, N=2 n+1$, and $n$ can be any non-negative integer.

\subsection{Linear decoding design}

In the aforementioned transmission model, the received signal given in (1) can be rewritten as

$$
\begin{aligned}
& \boldsymbol{V}_{1}=\left\{\prod_{k, l \in \mathcal{K} \backslash 1, k \neq l,(k, l) \neq(2,3)}\left(\left(\boldsymbol{T}_{23}\right)^{-1} \boldsymbol{T}_{k l}\right)^{n_{k l}} \mid \sum_{k, l \in \mathcal{K} \backslash 1, k \neq l,(k, l) \neq(2,3)} n_{k l} \leq m^{*}+1\right\}, \\
& \boldsymbol{V}_{3}=\left\{\left(\boldsymbol{T}_{23}\right)^{-1} \prod_{k, l \in \mathcal{K} \backslash 1, k \neq l,(k, l) \neq(2,3)}\left(\left(\boldsymbol{T}_{23}\right)^{-1} \boldsymbol{T}_{k l}\right)^{n_{k l}} \mid \sum_{k, l \in \mathcal{K} \backslash 1, k \neq l,(k, l) \neq(2,3)} n_{k l} \leq m^{*}\right\}, \\
& \boldsymbol{V}_{j}=\boldsymbol{H}_{1 j}^{-1} \boldsymbol{H}_{13} \boldsymbol{V}_{3} \text {, } \\
& \boldsymbol{T}_{k l}=\left(\boldsymbol{H}_{k 1}\right)^{-1} \boldsymbol{H}_{k l}\left(\boldsymbol{H}_{1 l}\right)^{-1} \boldsymbol{H}_{13} \text {. }
\end{aligned}
$$




$$
\boldsymbol{y}_{k}=\underbrace{\overline{\boldsymbol{H}}_{k}^{k} \boldsymbol{s}_{k}}_{\text {Desired subspace }}+\underbrace{\sum_{j \neq k} \overline{\boldsymbol{H}}_{j}^{k} \boldsymbol{s}_{j}}_{\text {Interference subspace }}+\boldsymbol{z}_{k},
$$

where $\overline{\boldsymbol{H}}_{j}^{k}=\boldsymbol{H}_{k j} \boldsymbol{V}_{j}$. We assume that the IA conditions are satisfied. Let $\overline{\boldsymbol{H}}_{I}^{k} \in \mathbb{C}^{N \times\left(N-d_{k}\right)}$ denotes the $N-d_{k}$ matrix spanning all interference subspaces, i.e., $\overline{\boldsymbol{H}}_{j}^{k}$ for $j \in$ $\{1, \cdots, K\}, j \neq k$ are all spanned by $\overline{\boldsymbol{H}}_{I}^{k}$. Before going further into the description, we introduce the following lemma:

Lemma 1. Let $\boldsymbol{A}_{1} \in \mathbb{C}^{N \times n}$ and $\boldsymbol{A}_{2} \in \mathbb{C}^{N \times n}(N>n)$, where $\operatorname{rank}\left(\boldsymbol{A}_{1}\right)=n$ and $\operatorname{rank}\left(\boldsymbol{A}_{2}\right)=m,(m \leq n)$ and $\operatorname{span}\left(A_{2}\right) \subset \operatorname{span}\left(A_{1}\right)$. Then, for every $s_{2} \in \mathbb{C}^{n \times 1}, \exists s_{1} \in$ $\mathbb{C}^{n \times 1}$ such that $A_{1} s_{1}=A_{2} s_{2}$.

Using Lemma 1 at the receiver $k$, the interference subspace from the $j$ th transmitter can be expressed in terms of $\overline{\boldsymbol{H}}_{I}^{k}$ as

$$
\overline{\boldsymbol{H}}_{j}^{k} \boldsymbol{s}_{j}=\overline{\boldsymbol{H}}_{I}^{k} \boldsymbol{s}_{j I} \text {, where } \boldsymbol{s}_{j I}=\left(\overline{\boldsymbol{H}}_{I}^{k^{H}} \overline{\boldsymbol{H}}_{I}^{k}\right)^{-1} \overline{\boldsymbol{H}}_{I}^{k^{H}} \overline{\boldsymbol{H}}_{j}^{k} \boldsymbol{s}_{j} .
$$

Substituting (6) into (5) yields

$$
\begin{aligned}
\boldsymbol{y}_{k} & =\overline{\boldsymbol{H}}_{k}^{k} \boldsymbol{s}_{k}+\overline{\boldsymbol{H}}_{I}^{k} \bar{s}_{k}+\boldsymbol{z}_{k} \\
& =\left[\begin{array}{ll}
\overline{\boldsymbol{H}}_{k}^{k} & \overline{\boldsymbol{H}}_{I}^{k}
\end{array}\right]\left[\left[\begin{array}{ll}
\boldsymbol{s}_{k}^{T} & \overline{\boldsymbol{s}}_{k}^{T}
\end{array}\right]^{T}+\boldsymbol{z}_{k}\right. \\
& =\boldsymbol{B}_{k} \tilde{\boldsymbol{s}}_{k}+\boldsymbol{z}_{k},
\end{aligned}
$$

where $\overline{\boldsymbol{s}}_{k}=\left(\boldsymbol{s}_{1 I}+\cdots+\boldsymbol{s}_{(k-1) I}+\boldsymbol{s}_{(k+1) I}+\cdots+\boldsymbol{s}_{K I}\right)$, $\boldsymbol{B}_{k} \in \mathbb{C}^{N \times N}$ is a full rank matrix that spans the union of the desired and the interference subspaces, and $\tilde{\boldsymbol{s}}_{k}$ is the $N \times 1$ vector consisting of the $d_{k}$ desired streams and the $N-d_{k}$ interference streams. Equation 7 gives the mathematical formulation of a linear determined decoding problem, where an $N$ length source data vector $\tilde{\boldsymbol{s}}_{k}$ is mixed by a constant mixing matrix $\boldsymbol{B}_{k}$ to produce a vector $\boldsymbol{y}_{k}$ of $N$ observations. Such a decoding problem can be resolved using classical criteria such as zero-forcing (ZF), minimum mean square error (MMSE), and maximum likelihood (ML).

\section{IA precoding subspaces optimization}

In this section, we aim to optimize the IA precoding subspaces in the scheme described above. From (3), it can be noted that the modified precoding matrices defined as

$$
\boldsymbol{V}_{k}=\boldsymbol{W} \boldsymbol{V}_{k}^{I A} \forall k \in \mathcal{K},
$$

where $V_{k}^{I A}$ is the original matrix derived with respect to the IA conditions and $W$ is any diagonal matrix, which satisfy the IA conditions. That is, the projection of all precoding matrices of the IA scheme on a common diagonal matrix $\boldsymbol{W}$ keeps the IA conditions respected.

The precoding subspaces can be optimized by judiciously selecting the components of $W$ in (8). This diagonal matrix $W$ determines the interference and the desired subspaces design, while maintaining the IA conditions at the receivers. We assume both MMSE- and ZF-based detection schemes, widely used due to the simplicity of their implementation, and we derive two different optimized designs that maximize the network sum rate in both cases.

\subsection{MMSE-based decoder - iterative solution}

Assuming an MMSE decoder, the mutual information between the $k$ th transmitter and its intended receiver $k$ can be expressed as [11]

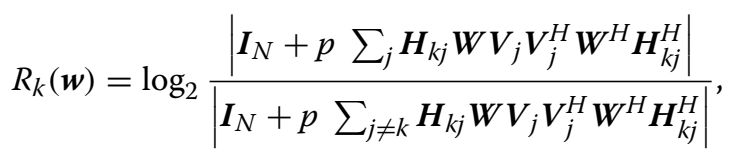

where $p$ is the user average transmit power over the average noise power assumed equal at all receivers. Using the Sylvester's determinant theorem [12], the fact that all channel matrices are diagonal, and the definition of $\boldsymbol{B}_{k}$ and $A_{k}$ as

$$
\begin{gathered}
\boldsymbol{B}_{k}=\sum_{j=1, j \neq k}^{K} \boldsymbol{H}_{k j} \boldsymbol{V}_{j} \boldsymbol{V}_{j}^{H} \boldsymbol{H}_{k j}^{H} \\
\boldsymbol{A}_{k}=\boldsymbol{B}_{k}+\boldsymbol{H}_{k k} \boldsymbol{V}_{k} \boldsymbol{V}_{k}^{H} \boldsymbol{H}_{k k}^{H},
\end{gathered}
$$

(9) can be reformulated in the following compact form as

$$
R_{k}(\tilde{\boldsymbol{w}})=\log _{2} \frac{\left|\boldsymbol{I}_{N}+p \tilde{\boldsymbol{W}} \boldsymbol{A}_{k}\right|}{\left|\boldsymbol{I}_{N}+p \tilde{\boldsymbol{W}} \boldsymbol{B}_{k}\right|},
$$

where $\tilde{\boldsymbol{W}}=\boldsymbol{W}^{H} \boldsymbol{W}$ is a diagonal matrix and its diagonal is defined as the vector $\tilde{\boldsymbol{w}}$ with positive components $\left(\tilde{w}_{i} \forall i\right)$. One can notice from (10) that matrices $\boldsymbol{A}_{k}$ and $\boldsymbol{B}_{k}$ are written as the sum of semi-definite positive matrices and hence are positive semi-definite. Consequently, the Cholesky decomposition ${ }^{\mathrm{b}}$ can be applied [13], and the matrices $\boldsymbol{A}_{k}$ and $\boldsymbol{B}_{k}$ can be rewritten as

$\boldsymbol{A}_{k}=\boldsymbol{L}_{A_{k}}^{H} \boldsymbol{L}_{A_{k}}$ with $\boldsymbol{L}_{A_{k}}^{H}=\left[\begin{array}{ll}\boldsymbol{L}_{B_{k}}^{H} & \boldsymbol{H}_{k k} \boldsymbol{V}_{k}\end{array}\right]$ and $\boldsymbol{B}_{k}=\boldsymbol{L}_{B_{k}}^{H} \boldsymbol{L}_{B_{k}}$.

Substituting (12) into (11) yields the $k$ th user rate

$$
R_{k}(\tilde{\boldsymbol{w}})=\log _{2} \frac{\left|\boldsymbol{I}_{N}+p \boldsymbol{L}_{A_{k}} \tilde{\boldsymbol{W}} \boldsymbol{L}_{A_{k}}^{H}\right|}{\left|\boldsymbol{I}_{N}+p \boldsymbol{L}_{B_{k}} \tilde{\boldsymbol{W}} \boldsymbol{L}_{B_{k}}^{H}\right|} .
$$


Our goal is to seek $\tilde{\boldsymbol{w}}$ that maximizes the total mutual information (i.e., network sum rate) in the IC under the constant total transmit power linear constraint. The maximization problem is then defined as

$$
\underset{\tilde{\boldsymbol{w}}}{\arg \max } \frac{1}{N} \sum_{k=1}^{K} \log _{2} \frac{\left|\boldsymbol{I}_{N}+p \boldsymbol{L}_{A_{k}} \tilde{\boldsymbol{W}} \boldsymbol{L}_{A_{k}}^{H}\right|}{\left|\boldsymbol{I}_{N}+p \boldsymbol{L}_{B_{k}} \tilde{\boldsymbol{W}} \boldsymbol{L}_{B_{k}}^{H}\right|},
$$

subject to the total transmit power constraint

$$
\sum_{k=1}^{K} \operatorname{tr}\left(\tilde{W} V_{k} \boldsymbol{V}_{k}^{H}\right)=K, \tilde{w}_{i} \geq 0, i \in\{1, \ldots, N\}
$$

It is not obvious whether a closed-form solution can be obtained or not; therefore, one can search for the solution iteratively. However, the convergence towards the global maximum is not guaranteed unless the objective function is concave. The proof of the concavity with respect to the variable vector $\tilde{\boldsymbol{w}}$, requires the objective function to be twice differentiable and its Hessian matrix to be negative semi-definite [14]. Indeed, a similar problem has been treated in [15] for the three-user IA scheme. The authors have demonstrated that a function having the form of (14) is concave if $\boldsymbol{A}_{k}$ and $\boldsymbol{B}_{k}$ are defined as in (12) (see Appendix 1 in [15]). In order to find the solution that achieves the optimum, we propose to use the projected gradient method with an optimized variable step size (details are given in Appendix 2). Other algorithms can also be used such as simple gradient descent method using Lagrange multipliers.

Remark 1. In [15], the diagonal matrix $W$ has been optimized under an MMSE receiver assumption. However, the study has been restricted to the three-user SISO interference channel case. Also, there is no description of the iterative algorithm used for achieving the solution. In this section, we have provided a general design for the $K$-user SISO interference channel case. We have also described an iterative method (see Appendix 2) to converge towards the solution.

\subsection{ZF-based decoder - closed-form solution}

In the previous subsection, we have proposed to optimize the precoding subspaces using iterative processing when an MMSE is applied at the receiver. In this section, we apply a ZF criterion at the receiver. Then, we propose a closed-form solution for $\boldsymbol{w}$ that is asymptotically optimal. This solution is obtained from the network sum-rate maximization problem approximation for very high SNR and under the hypothesis of a ZF applied at all receivers. It also avoids the need for a numerical solver that requires a matrix inversion at each iteration and increases the processing time and computational cost.
Assuming a ZF criterion at all receiver nodes and an IA design at all transmitters, the mutual information between the $k$ th transmitter and its intended receiver $k$ is expressed as

$$
R_{k}=\log _{2}\left|\boldsymbol{I}_{\boldsymbol{N}}+p \boldsymbol{U}_{k} \boldsymbol{H}_{k k} \boldsymbol{W} \boldsymbol{V}_{k} \boldsymbol{V}_{k}^{H} \boldsymbol{W}^{\boldsymbol{H}} \boldsymbol{H}_{k k}^{H} \boldsymbol{U}_{k}^{H}\right|
$$

where $\boldsymbol{U}_{k}$ is the interference canceler at the $k$ th receiver. Assuming well-conditioned channel matrices and using Sylvester's determinant theorem [12], the $k$ th user rate can be approximated for high SNR values by

$$
R_{k} \underset{\mathrm{SNR}>>1}{\approx} \log _{2}\left|p \boldsymbol{H}_{k k} \boldsymbol{W} \boldsymbol{V}_{k} \boldsymbol{V}_{k}^{H} \boldsymbol{W}^{H} \boldsymbol{H}_{k k}^{H} \boldsymbol{U}_{k}^{H} \boldsymbol{U}_{k}\right| \text {. }
$$

Now, we intend to maximize the sum-rate approximation $\sum_{k} R_{k}$ with respect to $w$ under the total transmit power constraint. Using the following equivalence:

$\underset{\tilde{\boldsymbol{w}}}{\arg \max } \sum_{k=1}^{K} R_{k}(\tilde{\boldsymbol{w}}) \equiv \underset{\tilde{\boldsymbol{w}}}{\arg \max }|\tilde{\boldsymbol{W}}|^{K} \prod_{k=1}^{K}\left|\boldsymbol{H}_{k k} \boldsymbol{V}_{k} \boldsymbol{V}_{k}^{H} \boldsymbol{H}_{k k}^{H}\right|\left|\boldsymbol{U}_{k}^{H} \boldsymbol{U}_{k}\right|$

and the fact that $\prod_{k=1}^{K}\left|\boldsymbol{H}_{k k} \boldsymbol{V}_{k} \boldsymbol{V}_{k}^{H} \boldsymbol{H}_{k k}^{H}\right|\left|\boldsymbol{U}_{k}^{H} \boldsymbol{U}_{k}\right|$ is positive, the optimization problem in (17) can also be reduced to

$$
\underset{\tilde{\boldsymbol{w}}}{\arg \max }|\tilde{\boldsymbol{W}}|,
$$

subject to $\sum_{k} \operatorname{tr}\left(\tilde{W} V_{k} V_{k}^{H}\right)=K N, \tilde{w}_{i}>0 \forall i$.

We notice that the problem in (18) is independent from the channel matrices, and the objective function is a simple determinant of a diagonal matrix, hence, a concave function. Introducing the Lagrange multiplier $\lambda$, the convex dual of this problem is formulated as follows [14]:

$$
\underset{\tilde{\boldsymbol{w}}}{\arg \max } \underset{\lambda}{\arg \min } L(\tilde{\boldsymbol{w}}, \lambda)
$$

where the Lagrangian function is defined as

$$
L(\tilde{\boldsymbol{w}}, \lambda)=|\tilde{\boldsymbol{W}}|-\lambda\left(\operatorname{tr}\left(\sum_{k} \tilde{\boldsymbol{W}} \boldsymbol{V}_{k} \boldsymbol{V}_{k}^{H}\right)-K N\right),
$$

with $\operatorname{tr}\left(\sum_{k} \tilde{\boldsymbol{W}} \boldsymbol{V}_{k} \boldsymbol{V}_{k}^{H}\right)=\sum_{i=1}^{N} c_{i} \tilde{w}_{i}$, and $c_{i}=\sum_{k=1}^{K}\left\|\boldsymbol{v}_{k i}\right\|^{2}$,

and $\boldsymbol{v}_{k i}$ stands for the $i$ th row of the matrix $\boldsymbol{V}_{k}$. Since the objective function is concave, the Karush Kuhn-Tucker (KKT) conditions are sufficient to determine the global optimum. The KKT conditions of the problem in (19) are given by

$$
\begin{aligned}
\left.\nabla_{\tilde{\boldsymbol{w}}} L(\tilde{\boldsymbol{w}}, \lambda)\right|_{\tilde{\boldsymbol{w}}=\tilde{\boldsymbol{w}} *} & =0, \lambda>0, \\
\sum_{i=1}^{N} c_{i} \tilde{w}_{i} & =K N
\end{aligned}
$$

The solution of the linear problem in (21) is obtained as

$$
\tilde{w}_{i}^{*}=\frac{K}{c_{i}}, i \in\{1, \ldots, N\} \text {. }
$$


Hence, the components of $\boldsymbol{w}$ are obtained as $w_{i}^{*}=\sqrt{\tilde{w}_{i}^{*}}$ for all $i$. It is worth noting that beside maximizing the sumrate, the problem of maximizing the individual rate using the approximation in (16) has the same solution obtained in (22).

A major advantage of the proposed solution is the fact that it has an analytic simple expression making its implementation complexity very low. Indeed, the other algorithms proposed for sum-rate maximization and interference power minimization in SISO and $\mathrm{MIMO}^{\mathrm{c}}$ transmissions achieve the optimum using singular value decomposition (SVD) [11] and/or an iterative algorithm that requires hundreds to thousands iterations to converge [7-9,16].

\subsection{Complexity and sum-rate performance}

The computational complexity is a major bottleneck of practical implementation that is considered in system designs. In the following, we discuss the complexity of the precoding schemes proposed above.

The first optimized design that maximizes the sum-rate assuming an MMSE detector is obtained using the projected gradient descent method. This iterative method requires at each iteration the computational cost of the first-order derivative of the objective function. Looking at the expression given in (34), one can notice that the derivative is calculated using matrix multiplications and matrix inversions with dimensions $N \times N$. Therefore, the computational complexity at each iteration can be considered of order $\mathcal{O}\left(N^{3}\right)$. On the other hand, the design based on a closed-form solution requires the computation of a Frobenius norm and $N$ real division. Thus, the complexity order is $\mathcal{O}(N)$.

Figure 3 illustrates the average sum-rate per dimension performance of the non-optimized design proposed by Choi et al. in [6] and the optimized designs W-MMSE and W-ZF, which represent the designs based on the solutions to the problems given in (14) and (18), where we optimize the precoding subspaces of the IA scheme assuming an MMSE and a ZF, respectively. The optimizations are applied to the IA design proposed by Choi et al. It can be observed that for $N=3$ and $N=7$, the W-MMSE design outperforms the W-ZF design with the closedform solution in the low SNR region. However, when the SNR becomes very high, the sum-rate performance for both designs get very close. This is due to the validity of the approximation in (16) for high SNR. On the other hand, the resulting gain of the optimized designs over the nonoptimized one is significant, e.g., at $20 \mathrm{~dB}$ the gain is between 4 and 5 bits $/ \mathrm{s} / \mathrm{Hz} / \mathrm{dim}$.

It is important to note that the proposed designs result from the optimization of the original designs proposed in [6] and not the optimal IA design that maximizes the sumrate. It explains why, when we compare the designs for different dimensions $N$, a higher sum-rate is obtained for $N=3$ compared to the design for $N=7$. Furthermore, in order to have a fair comparison between the cases $N=3$ and $N=7$, the average sum rate performance is normalized by the dimension $N$, which means that the sum rate is divided by the number of symbols in one symbol vector as shown in (14).

\section{Precoding vectors design within IA subspaces}

The previous section has addressed the optimization of the IA precoding subspaces at once using a diagonal matrix $W$. However, there was no claim for the optimality of the precoding vectors within IA subspaces. In this section, we propose to maintain the IA subspaces design at the transmitters, and we aim to optimize the precoding vectors within each subspace. We consider both cases: MMSE and ZF criterion at the receiver, and we attempt to maximize the network sum-rate in each case.

The precoding matrices defined in (4) are of size $N \times d_{k}$ with $N>d_{k}, \forall k$. Thus, introducing a new combination matrix $C_{k} \in \mathbb{C}^{d_{k} \times d_{k}}$ at each transmitter node as follows

$$
\boldsymbol{P}_{k}=\boldsymbol{V}_{k} \boldsymbol{C}_{k}, \forall k \in \mathcal{K},
$$

will modify the basis of $V_{k}$ within its own subspace without modifying the subspace itself. These variables can later be defined taking into account different criteria such as MSE, BER, sum-rate, and average transmit power. Next, we show how to optimize the additional combination matrices so as to maximize the network sum-rate.

\subsection{MMSE-based decoder}

Assuming an MMSE at all receivers, the mutual information between the $k$ th transmitter and its dedicated receiver $k$ can be written as a function of the combination matrices $C_{k} \forall k$ as follows

$$
R_{k}=\log _{2} \frac{\left|\boldsymbol{I}_{N}+p \sum_{j=1}^{K} \boldsymbol{H}_{k j} \boldsymbol{P}_{j} \boldsymbol{P}_{j}^{H} \boldsymbol{H}_{k j}^{H}\right|}{\left|\boldsymbol{I}_{N}+p \sum_{j \neq k}^{K} \boldsymbol{H}_{k j} \boldsymbol{P}_{j} \boldsymbol{P}_{j}^{H} \boldsymbol{H}_{k j}^{H}\right|} .
$$

Now, in order to maximize the sum-rate under the individual transmit power constraint, we propose the following maximization problem

$$
\begin{aligned}
& \underset{\boldsymbol{C}_{k}, k \in \mathcal{K}}{\arg \max } \frac{1}{N} \sum_{k=1}^{K} \log _{2} \frac{\left|\boldsymbol{I}_{N}+p \sum_{j=1}^{K} \overline{\boldsymbol{H}}_{k j} \boldsymbol{C}_{j}\left(\overline{\boldsymbol{H}}_{k j} \boldsymbol{C}_{j}\right)^{H}\right|}{\left|\boldsymbol{I}_{N}+p \sum_{j \neq k}^{K} \overline{\boldsymbol{H}}_{k j} \boldsymbol{C}_{j}\left(\overline{\boldsymbol{H}}_{k j} \boldsymbol{C}_{j}\right)^{H}\right|} \\
& \text { subject to } \operatorname{tr}\left(\boldsymbol{V}_{k} \boldsymbol{C}_{k} \boldsymbol{C}_{k}^{H} \boldsymbol{V}_{k}^{H}\right)=N, \forall k \in \mathcal{K} .
\end{aligned}
$$

where $\overline{\boldsymbol{H}}_{k j}=\boldsymbol{H}_{k j} \boldsymbol{V}_{j}$. It is well-known that the optimal solution is the one that nullifies the gradient of the sumrate expression. However, a closed-form solution is not obvious due to a complicated expression of the first-order derivative as shown in (32) in Appendix 1. Therefore, we 


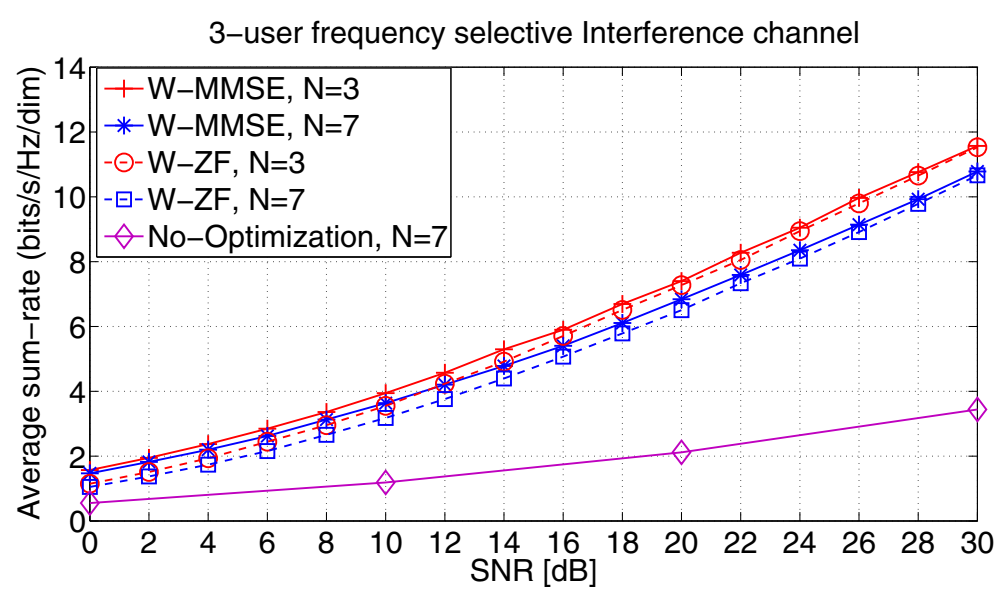

Figure 3 Average sum rate per dimension of the two proposed designs for subspace improvement when $N=3$ and $N=7$.

attempt to get close to the solution iteratively. We use an iterative algorithm that optimizes the cost function with respect to one variable while the others remain fixed. In our reasoning, each variable is considered as one of the precoding matrices. This technique results in a nonconvex optimization due to the dependence between the precoding matrices. At each iteration, the optimization is based on the gradient descent widely used in MIMO multiuser channels. The iterative algorithm for the sum-rate maximization is detailed in Algorithm 1.

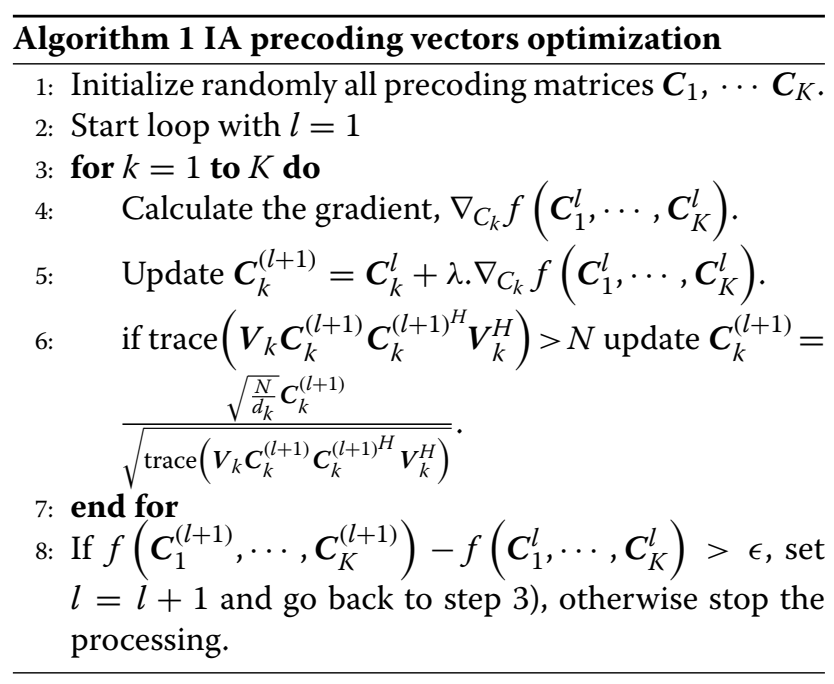

In this algorithm the gradient is defined in (32) in Appendix 1, $f$ describes the objective function given in (25), and the precoding matrices are supposed to be of unit Frobenius norm. The step size $\lambda$ is updated using the backtracking search, which is an effective and quite simple method [14]. Despite the non-convexity of the multi-variable objective function, as long as the variable is steered in the gradient direction, the algorithm converges to a local maximum. In our simulations, the convergence of this iterative algorithm is supposed to be achieved either when

$$
\sum_{k}\left\|\nabla_{C_{k}^{(l)}} R\right\|<\epsilon
$$

or when a maximum number of iterations is reached, and $\epsilon$ is defined as a tolerance value. In our simulations, we assume $\epsilon=10^{-2}$.

\subsection{ZF-based decoder}

Given the $k$ th user rate, the ZF-based detector uses a matrix $\boldsymbol{U}_{k}$ to cancel the interference, yielding an equivalent $d_{k} \times d_{k}$ MIMO transmission model. Many options exist to find the best family of combination matrices $\left\{\boldsymbol{C}_{j}\right\}$ in order to maximize the sum-rate. The channel model after interference suppression at receiver $k$ is obtained as

$$
\begin{aligned}
\boldsymbol{y}_{k} & =\boldsymbol{U}_{k} \boldsymbol{H}_{k k} \boldsymbol{V}_{k} \boldsymbol{C}_{k} \boldsymbol{x}_{k}+\boldsymbol{U}_{k} \boldsymbol{z}_{k}, \\
& =\tilde{\boldsymbol{H}}_{k} \boldsymbol{C}_{k} \boldsymbol{x}_{k}+\boldsymbol{U}_{k} \boldsymbol{z}_{k},
\end{aligned}
$$

where $\boldsymbol{U}_{k}$ is the decoding matrix at the $k$ th receiver. It is defined as the $d_{k} \times N$ interference null space. The model defined in (27) is a typical MIMO single user model with channel matrix $\tilde{\boldsymbol{H}}_{k}$ and precoding matrix $\boldsymbol{C}_{k}$. One optimized form of $\boldsymbol{C}_{k}$ is the one composed of the right singular vector of the new channel matrix $\tilde{\boldsymbol{H}}_{k}$. Such a precoding scheme achieves the channel capacity as described in [17]. Another form that requires less computational complexity is the one that orthonormalizes the columns of the original precoding matrix $V_{k}$. In [18], the authors have shown that this last form gets close to the maximum information rate when the SNR becomes high. 


\subsection{Complexity and sum-rate performance}

The algorithm that optimizes the solution iteratively in Subsection 5.1 is based on the gradient descent method. At each iteration, the iterative algorithm requires the gradient of the objective function that needs itself inversion of $N \times N$ full rank matrices. Thus, the total computational complexity depends mainly on the number of iterations and on the precoding matrices dimensions. The complexity cost is of order $\mathcal{O}\left(n b_{i} N^{3}\right)$ where $n b_{i}$ is the number of iterations.

Figure 4 illustrates the sum-rate per dimension of the proposed design that uses Algorithm 1 and the design OW-ZF that orthonormalizes the precoding vectors. For the subspace optimization of OW-ZF, we use the closed form solution derived in Subsection 4.2. One can observe a sum-rate performance loss in the case of OW-ZF compared to the CW-MMSE for low SNR values, whereas when the SNR increases, both sum rates become very close. However, the OW-ZF requires less operations, the complexity cost is of order $\mathcal{O}\left(N d_{k}^{2}\right)$ at each transmitter, and no joint processing is required for the optimization design.

Remark 2. The proposed optimization of IA precoding subspaces cannot directly be extended to MIMO interference channels, as the channel matrices are no longer diagonal in the MIMO system model. However, the proposed optimization of the precoding vectors within each IA subspace can be used for MIMO optimization design since the IA conditions at all receiver are always maintained.

\section{Convergence rate of the iterative solutions}

In Subsections 4.1 and 5.1, we have proposed two iterative solutions, one aims to optimize the IA subspaces and the other optimizes the precoding vectors within each IA subspace without modifying the subspace itself.

The first iterative solution to the problem in (14) for the IA subspaces optimization is reached using the projected gradient method. We have mentioned that the objective function is concave, thus, the convergence towards the global optimum is guaranteed. On the other hand, the iterative solution proposed for the IA precoding vector optimization is reached using an algorithm based on the gradient descent method for a multi-variable objective function. Thereby, the objective function changes at every iteration yielding a nonconvex optimization problem. However, as long as the iterative method is based on the gradient descent and the variable follows the direction of the gradient using an optimized step size, a convergence towards a local optimum is guaranteed.

The convergence rates of the discussed iterative solutions above are shown in Figure 5. For the projected gradient descent method, the convergence towards either the optimal solution or a neighboring optimal solution requires hundreds of iterations. This slow convergence rate diverts the attention to the closed-form solution obtained in Subsection 4.2. Now, looking at the design for precoding vector optimization within their subspace, the convergence rate seems fast. For example, almost 10 to 15 iterations are required to achieve a near-optimal value at 15 and $25 \mathrm{~dB}$ when $N=7$. This fast convergence implies that for small dimensions and for precoding vector optimization as discussed in Subsection 5.2, the computational complexity is not that far from other proposed designs.

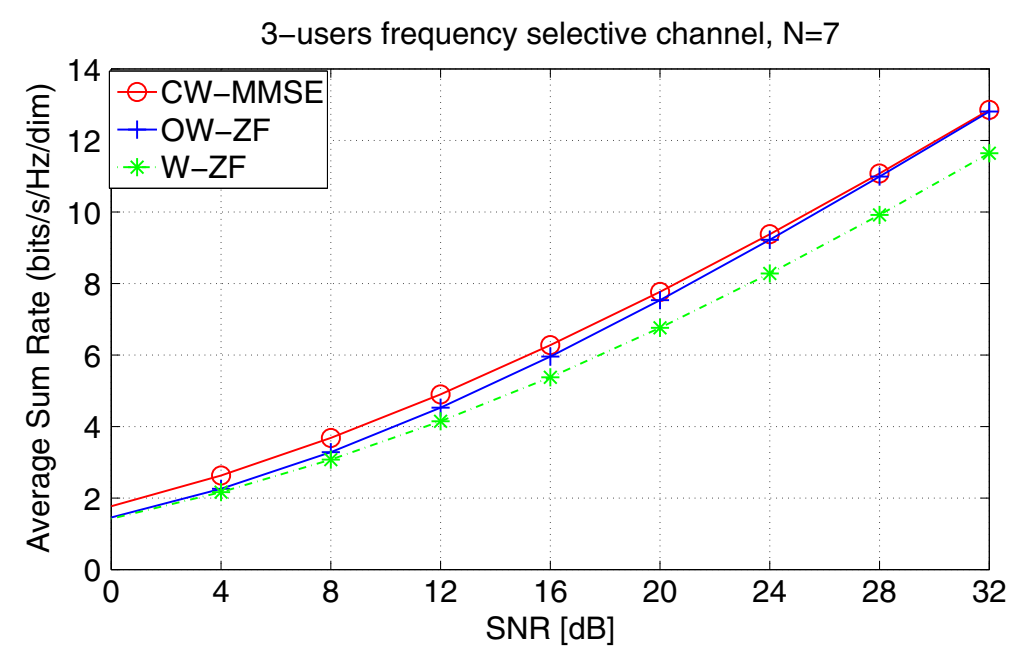

Figure 4 Average sum rate per dimension of the proposed precoding vectors design using the combination matrices. When $N=3$ and $N=7$. 

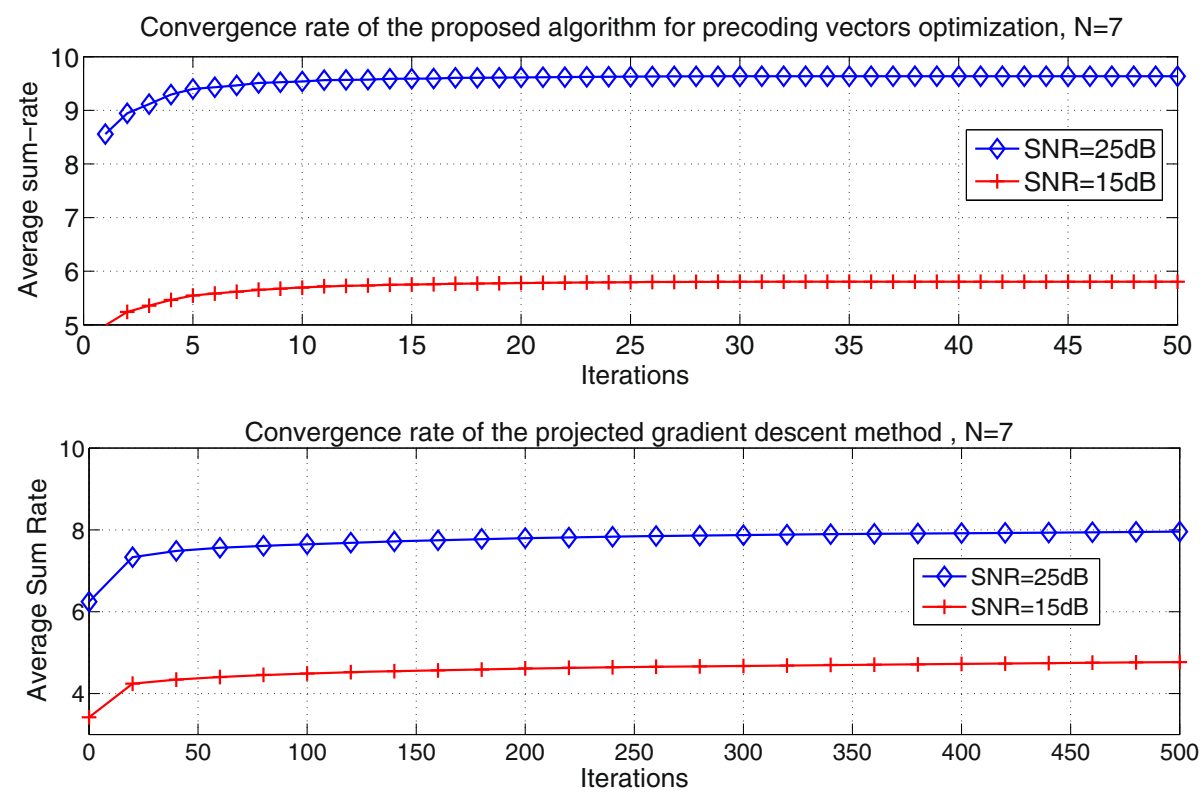

Figure 5 Convergence of the iterative algorithm in Section 5.1 and the projected gradient method in Subsection 4.1.

\section{Comparison of the proposed optimized designs to the state of art schemes}

In this section, we compare the proposed designs to the distributed designs proposed in $[7,8]$ in terms of sumrate per dimension. We consider a three-user frequencyselective SISO IC, with the model proposed in Section 2. The total independent stream number from all users is equal to $T=3 n+1$, and the precoding vectors length is $N=2 n+1$ for all users, and $n$ can be any nonnegative number. The transmit constellation is Gaussian continuously distributed, and the channel coefficients are circularly symmetric complex Gaussian distributed with zero mean and unit variance. The following abbreviations are used for the compared designs:

- OW-ZF : the proposed IA design with the closed-form solution derived in Subsection 4.2 that uses orthogonal precoding vectors

- CW-MMSE: the IA design with the two iterative proposed optimization in Subsections 4.1 and 5.1

- IA-Iter: the IA design obtained with the distributed algorithm proposed in [8]

- Max-SINR: the beamforming design proposed in [7] that maximizes the signal-to-interference-and-noise ratio (SINR) of all streams

Figures 6, 7 and 8 illustrate the average sum-rate per dimension performance of the OW-ZF, the CW-MMSE, the IA-Iter, and the Max-SINR for $N=3, N=5$, and $N=$ 7 , respectively. Compared to the CW-MMSE, the OWZF performs similarly for all configurations with a slight loss for low and medium SNR values. This is due to the fact that a ZF criterion becomes equivalent to an MMSE when the SNR becomes very high, and that the OW-ZF design is based on an approximation for high SNR. On the other hand, an important gain is obtained over the IA-Iter design over the whole SNR region when $N=3$ and $N=5$, e.g., at $20 \mathrm{~dB}$ a gain of about 2.2 to $2.5 \mathrm{bits} / \mathrm{s} / \mathrm{Hz}$ and 1.7 to $2 \mathrm{bits} / \mathrm{s} / \mathrm{Hz}$ is obtained for $N=3$ and $N=5$, respectively. It is worth noting that in addition to this gain, the OW-ZF design is a closed-form, thus, it exhibits a much less computational complexity than the other designs. It also does not require any iterative processing to achieve the solution, which can sustain the complexity order when $N$ increases. Now, considering the beamforming optimization design that maximizes the SINR referred to as Max-SINR, this latter outperforms the proposed designs in the low and medium SNR region. However, this resulting gain decreases as the SNR increases in the medium to high SNR region. For example, the OW-ZF design and the Max-SINR design reach the same sum-rate value of about $10.4 \mathrm{bits} / \mathrm{s} / \mathrm{Hz}$ at $30 \mathrm{~dB}$ when $N=3$. This result can show that in some particular cases, the proposed designs are very close to one of the most efficient designs when the SNR is high enough while keeping a low complexity level such as the OW-ZF design.

On the other side, compared to the orthogonal interference mitigation techniques such as time division multiple access (TDMA), the optimized IA scheme (i.e., CWMMSE) achieves similar performance in the low SNR region. However, when the SNR increases, i.e., beyond $15 \mathrm{~dB}$, the gain between both optimized IA designs (i.e., OW-ZF and CW-MMSE) and the TDMA starts to get 

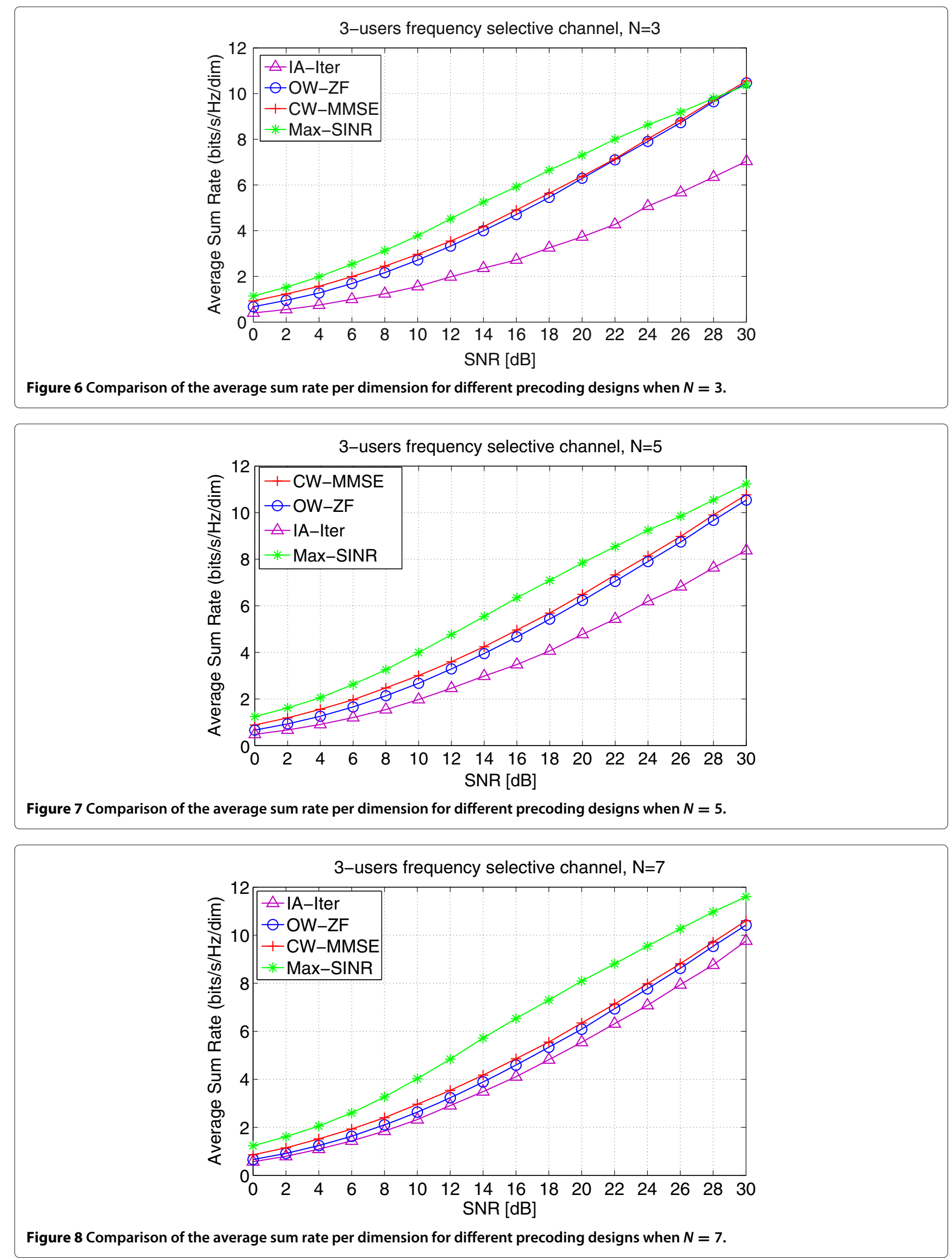


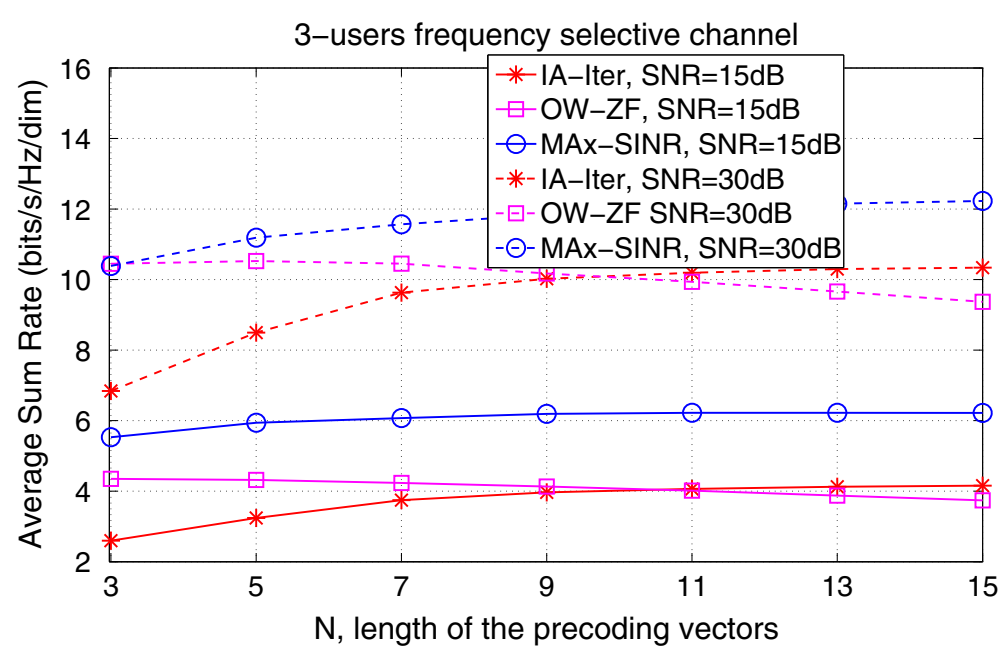

Figure 9 Evolution of the average sum rate per dimension with the precoding vector length.

wider, and achieves $2 \mathrm{bits} / \mathrm{s} / \mathrm{Hz} / \mathrm{dim}$ over the TDMA at 30 $\mathrm{dB}$. This is due to the suboptimality of the IA design in the low SNR region and its tendency to optimality in the high SNR region [4].

Next, Figure 9 evaluates the performance of the following designs: OW-ZF, IA-Iter, and Max-SINR, as a function of the precoding vectors sizes. At 15 and $30 \mathrm{~dB}$, the OWZF outperforms the IA-Iter for $N \leq 9$ and $N \leq 11$. On the other hand, it can be observed that the two iterative designs IA-Iter and Max-SINR result in an increasing sum-rate with the vector sizes. However, the closed-form design OW-ZF results in a decreasing sum-rate with the vectors sizes. This means that OW-ZF is close to the optimal for small precoding dimensions and starts moving away when $N$ increases.

\section{Conclusions}

In this paper, we have introduced three optimized designs for the IA scheme in a $K$-user SISO IC. The first and the second try to optimize the precoding subspaces at the IA transmitters through a common diagonal matrix assuming an MMSE and ZF linear detector, respectively. The third assumes an MMSE linear detector and seeks the optimal precoding vectors within a predefined subspace at each transmitter. The first and the third designs referred to as W-MMSE and C-MMSE, respectively, require iterative algorithms to converge to their optimum, whereas the second design referred to as W-ZF is obtained from a closed-form solution. Comparing to other IA distributed designs, the proposed designs show a significant sum-rate performance improvement and much less computational complexity when the closed-form solution is applied. To enhance the sum-rate performance, we have introduced an orthogonalization of the precoding vectors in the
W-ZF design, which enables to achieve a trade-off between complexity and data rate.

\section{Endnotes}

${ }^{a}$ This hypothesis is very optimistic, but it is taken by many research works in the literature.

${ }^{b}$ It is important to note that the Cholesky decomposition, originally defined for a positive definite matrix, can be extended to the positive semi-definite case.

${ }^{\mathrm{c}}$ The IA schemes proposed for MIMO transmission can also be used in SISO systems.

\section{Appendix 1}

Using the $k$ th information rate expression in (24), the sum rate can be written as

$$
R \equiv \sum_{k=1}^{K} \log _{2}\left|\boldsymbol{X}_{k}\right|-\log _{2}\left|\boldsymbol{Y}_{k}\right|
$$

where

$$
\begin{aligned}
\boldsymbol{X}_{k} & =\boldsymbol{I}+p \sum_{j=1}^{K} \overline{\boldsymbol{H}}_{k j} \boldsymbol{C}_{j}\left(\overline{\boldsymbol{H}}_{k j} \boldsymbol{C}_{j}\right)^{H} \\
\text { and } \boldsymbol{Y}_{k} & =\boldsymbol{I}+p \sum_{j \neq k}^{K} \overline{\boldsymbol{H}}_{k j} \boldsymbol{C}_{j}\left(\overline{\boldsymbol{H}}_{k j} \boldsymbol{C}_{j}\right)^{H}
\end{aligned}
$$

Since the sum-rate is real valued function and $C_{k} \forall k$ are complex variables, the gradient of the sum-rate can be calculated using the differential with respect to $C_{k}$. It is 
known to be $\mathrm{d} R=2 \partial R / C_{k}^{*}$. Details are given in [19]. Using the differential of $\log _{2}\left|X_{k}\right|$ computed as

$$
\begin{aligned}
\mathrm{d} \log _{2}\left|\boldsymbol{X}_{k}\right| & =\operatorname{trace}\left(\boldsymbol{X}_{k}^{-1} d \boldsymbol{X}_{k}\right), \\
\text { and } \mathrm{d} \boldsymbol{X}_{k} & =p \overline{\boldsymbol{H}}_{k j} \boldsymbol{C}_{j} \mathrm{~d} \boldsymbol{C}_{j}^{H} \overline{\boldsymbol{H}}_{k j}^{H},
\end{aligned}
$$

Using the following properties: $\operatorname{trace}\left(\boldsymbol{A} \mathrm{d} \boldsymbol{B}^{H}\right)=$ trace $\left(\boldsymbol{A}^{T} \mathrm{~d} \boldsymbol{B}^{*}\right), \mathrm{d}[\operatorname{trace}(\boldsymbol{A})]=\operatorname{trace}(\mathrm{d} \boldsymbol{A}), \operatorname{vec}(\mathrm{d} \boldsymbol{A})=\operatorname{dvec}(\boldsymbol{X})$, and trace $\left.\left(\boldsymbol{A}^{T} \boldsymbol{B}\right)=\operatorname{vec}(\boldsymbol{A})^{T}\right) \operatorname{vec}(\boldsymbol{B})$, and referring to [19] that describes the first-order differential and the Jacobian matrix properties, we obtain

$$
\mathrm{d} \log _{2}\left|\boldsymbol{X}_{k}\right|=\frac{2 p}{\ln 2} \operatorname{vec}\left(\overline{\boldsymbol{H}}_{k k}^{H} \boldsymbol{X}_{k}^{-1} \overline{\boldsymbol{H}}_{k k} \boldsymbol{C}_{k}\right)^{T} \cdot \operatorname{vec}\left(\mathrm{d} \boldsymbol{C}_{k}^{*}\right) .
$$

Thus, the gradient of $R$ w.r.t. $C_{k}^{*}$ is obtained as follows:

$$
\nabla_{\boldsymbol{C}_{k}^{(l)}} R=\frac{2 p}{\ln 2} \sum_{i=1}^{K} \overline{\boldsymbol{H}}_{i k}^{H} \boldsymbol{X}_{i}^{-1} \overline{\boldsymbol{H}}_{i k} \boldsymbol{C}_{k}-\frac{2 p}{\ln 2} \sum_{i \neq k}^{K} \overline{\boldsymbol{H}}_{i k}^{H} \boldsymbol{Y}_{i}^{-1} \overline{\boldsymbol{H}}_{i k} \boldsymbol{C}_{k} .
$$

\section{Appendix 2}

The projected gradient algorithm requires firstly the computation of the gradient with respect to $\tilde{\boldsymbol{w}}$

$$
\frac{\partial R(\tilde{\boldsymbol{w}})}{\partial \tilde{w}_{i}}=\sum_{k=1}^{K}\left(\boldsymbol{X}_{k i}-\boldsymbol{Y}_{k i}\right),
$$

where $\boldsymbol{X}_{k i}$ and $\boldsymbol{Y}_{k i}$ are defined as

$$
\begin{aligned}
& \boldsymbol{X}_{k i}=\frac{p}{N} \boldsymbol{l}_{A k i}\left(\boldsymbol{I}_{N}+p \boldsymbol{L}_{A k} \tilde{\boldsymbol{W}} \boldsymbol{L}_{A k}{ }^{H}\right)^{-1} \boldsymbol{l}_{A k i}^{H} \\
& \boldsymbol{Y}_{k i}=\frac{p}{N} \boldsymbol{l}_{B k i}\left(\boldsymbol{I}_{N}+p \boldsymbol{L}_{B k} \tilde{\boldsymbol{W}} \boldsymbol{L}_{B k}{ }^{H}\right)^{-1} \boldsymbol{l}_{B k i}^{H}
\end{aligned}
$$

with $\boldsymbol{l}_{A k i}$ and $\boldsymbol{l}_{B k i}$ are the $i$ th rows of the matrices $\boldsymbol{L}_{A k}$ and $\boldsymbol{L}_{B k}$, respectively. The constraint, defined in (5), can be formulated as

$$
\sum_{k=1}^{K} \operatorname{tr}\left[\boldsymbol{V}_{k} \tilde{\boldsymbol{W}} \boldsymbol{V}_{k}^{H}\right]=\sum_{i=1}^{N} \tilde{w}_{i} c_{i}
$$

with $c_{i}$ is the $i$ th component of the vector $c, c_{i}=\sum_{k}\left\|v_{k i}\right\|^{2}$, and $\boldsymbol{v}_{k i}$ is the $i$ th row of the matrix $\boldsymbol{V}_{k}$.

Equation 35 defines the set of $\tilde{\boldsymbol{w}}$ that satisfies the constraint, thus, given the gradient, we project it on the constraint hyperplane and update $\tilde{\boldsymbol{w}}$ by

$$
\tilde{\boldsymbol{w}}^{l+1}=\tilde{\boldsymbol{w}}^{l}+\mu \cdot \boldsymbol{p}_{\text {proj }}\left(\tilde{\boldsymbol{w}}^{l}\right),
$$

where $\mu$ is a variable step size and $\boldsymbol{p}(\tilde{\boldsymbol{w}})$ is the projected gradient defined as

$$
\boldsymbol{p}_{\text {proj }}\left(\tilde{\boldsymbol{w}}^{l}\right)=\nabla_{\boldsymbol{w}} R\left(\tilde{\boldsymbol{w}}^{l}\right)-\left(\boldsymbol{c}^{t} \nabla_{\boldsymbol{w}} R\left(\tilde{\boldsymbol{w}}^{l}\right)\right) \cdot \frac{\boldsymbol{c}}{\|\boldsymbol{c}\|^{2}} .
$$

The convergence towards the steady state is achieved either when

$$
\left\|\boldsymbol{p}\left(\tilde{\boldsymbol{w}}^{l}\right)\right\|<\epsilon
$$

with $\epsilon$ is the tolerance factor for stopping the iterations or a maximum number of iterations is attained. In this algorithm, the step size $\mu$ is a determining factor to ensure a faster convergence, thus, it must be judiciously selected. In [14], two line search methods are proposed: exact line search and inexact line search methods. In practice, most line searches are inexact, and many methods have been proposed. One is the backtracking method, which is employed for our design. It is very simple to implement and quite effective. Besides, the step size is updated at each iteration to satisfy $\tilde{w}_{i}>0$ for all $i$.

\section{Competing interests}

The authors declare that they have no competing interests.

\section{Acknowledgement}

The authors would like to thank the 'Institut Carnot Télécom et Société Numérique' for funding the work of this paper.

Received: 16 October 2013 Accepted: 13 April 2014 Published: 15 May 2014

\section{References}

1. H Sato, On degraded Gaussian two-user channels. IEEE Trans. Inform. Theory IT-24, 637-640 (1978)

2. A Carleial, Interference channels. IEEE Trans. Inform. Theory $\mathbf{2 4 , 6 0 - 7 0}$ (1978)

3. M Maddah-Ali, AS Motahari, AK Khandani, Communication over MIMOX channels: interference alignment, decomposition, and performance analysis. IEEE Trans. Inform. Theory 54(8), 3457-3470 (2008)

4. VR Cadambe, SA Jafar, Interference alignment and degrees of freedom of the K-user interference channel. IEEE Trans. Inform. Theory 54(8), 3425-3441 (2008)

5. AS Motahari, SO Gharan, MA Maddah-Ali, AK Khandani, Real interference alignment: exploiting the potential of single antenna systems. arXiv:0908.2282

6. SW Choi, SA Jafar, S-Y Chung, On the beamforming design for interference alignment. IEEE Commun. Lett. 13(11), 847-849 (2009)

7. K Gomadam, VR Cadambe, SA Jafar, Approaching the capacity of wireless networks through distributed interference alignment, in Proc. of IEEE Global Communications Conference, GLOBECOM (IEEE New Orleans, USA, 2008)

8. SW Peters, RW Heath, Interference alignment via alternating minimization, in Proc. of IEEE International Conference on Acoustics, Speech and Signal Processing, ICASSP, (2009), pp. 2445-2448

9. I Santamaria, O Gonzalez, R Heath, S Peters, Maximum sum-rate interference alignment algorithms for MIMO channels, in Proc. of IEEE Global Communications Conference, GLOBECOM (IEEE Miami, USA, 2010)

10. C Yetis, T Gou, SA Jafar, AH Kayran, On feasibility of interference alignment in MIMO interference networks. IEEE Trans. Signal Process. 58(9), 4771-4782 (2010)

11. H Sung, S Park, K Lee, I Lee, Linear precoder designs for K-user interference channels. IEEE Trans. Wireless Commun. 9(1), 291-300 (2010)

12. D Serre, Matrices: Theory and Applications, Second Edition. (Springer, New York, 2010)

13. NJ Higham, Analysis of the Cholesky Decomposition of a Semi-Definite Matrix. (Oxford University Press, 1990), pp. 161-185

14. S Boyd, L Vandenberghe, Convex Optimization. (Cambridge University Press, New York, 2004) 
15. D Kim, M Torlak, Optimization of interference alignment beamforming vectors. IEEE J. Selected Areas Commun. 28(9), 1425-1434 (2010)

16. H Shen, B Li, M Tao, Y Luo, The new interference alignment scheme for the MIMO interference channel, in Wireless Communications and Networking Conference (WCNC), 2010 IEEE (Sydney, Australia, 2010), pp. 1-6

17. PW Wolniansky, GJ Foschini, G Golden, RA Valenzuela, V-blast: an architecture for realizing very high data rates over the rich-scattering wireless channel, in Signals, Systems, and Electronics, 1998. ISSSE 98. 1998 URSI International Symposium On (IEEE Pisa, 1998), pp. 295-300

18. M Shen, A Host-Madsen, J Vidal, An improved interference alignment scheme for frequency selective channels, in Proc. of IEEE International Symposium on Information Theory, ISIT (Toronto, ON, 2008), pp. 6-11

19. J Magnus, H Neudecker, Matrix Differential Calculus with Applications in Statistics and Econometrics. (Wiley, Chichester, revised version 2007)

doi:10.1186/1687-1499-2014-79

Cite this article as: Fadlallah et al.: Interference alignment for a multi-user SISO interference channel. EURASIP Journal on Wireless Communications and Networking 2014 2014:79.

\section{Submit your manuscript to a SpringerOpen ${ }^{\circ}$ journal and benefit from:}

- Convenient online submission

- Rigorous peer review

- Immediate publication on acceptance

- Open access: articles freely available online

- High visibility within the field

- Retaining the copyright to your article

Submit your next manuscript at $>$ springeropen.com 